\title{
Mechatronic Assistance for Surgical Applications
}

\section{Gernot Kronreif}

\author{
Austrian Center for Medical Innovation and Technology \\ ACMIT Gmbh \\ Viktor Kaplan-Strasse 2, A-2700 Wiener Neustadt, Austria \\ e-mail: Gernot.Kronreif@acmit.at \\ www.acmit.at
}

\begin{abstract}
Medical Robotics is an interdisciplinary field that focuses on developing electromechanical devices for diagnosis and therapy. The long-term goal of this application area is to enable new medical techniques by providing new capabilities to the physician or by providing assistance during surgical procedures. The field has tremendous potential for improving the precision and capabilities of physicians when performing surgical procedures, and thus it is believed that the field will continue to grow. On the other hand - and unlike the area of factory robotics, which grew rapidly during the 1970s and 1980s - Medical Robotics has not yet gained widespread acceptance. There are still many technological challenges and research topics - including both the development of system components and the development of systems as a whole. The paper aims to give a short overview on the state-of-the-art of Medical Robotics in selected application areas, to highlight some open problems e.g., system architecture, user interfaces and safety issues, and finally to show some examples for medical robotic developments from Austria.
\end{abstract}

Keywords: Medical Robotics; Computer-Aided Surgery; Surgical Mechatronic Assistance

\section{Introduction}

The field of Medical Robotics is relatively new, with the first recorded medical application of a robot - i.e., image-guided orientation of a needle guide for biopsy of the brain - occurring in 1985 [1]. About the same time, research groups in Asia, Europe, and the USA began investigating other medical applications of robotics [2]. Currently, there are a large number of research laboratories, but only a few commercial ventures active in the field of Medical Robotics. After a short period of recovery from first negative experience, the topic once again is growing rapidly and dedicated sessions on Medical Robotics can now be found at almost any medical related conference. 
Compared to many other fields of medical technology, medical robots are still in their infant state and many research questions are still open for investigation and improvement of the technology. Based on the experience of previous research and clinical practice, an appropriate operating concept is essential for the success of such a system. In the context of minimally invasive procedures, current robots quite often are under fully control of surgeons by using a tele-operation setup in which the human operator manipulates a master input device and patient-side robot follows the input [9]. With such a setup, the robot allows the surgeon to have dexterity inside the body of the patient, scale down motions from normal human dimensions down to very small distances, and filter out tremor. The next generation of such robots will provide with a more intuitive connection between the operator and the instrument tips, with physical enhancements such as "no-fly" zones around delicate anatomical structures as well as better recognition of surgical motions and patient state to evaluate performance and predict health outcomes. In parallel, more autonomy will be implemented in such surgical assistants - e.g., in order to take over routine tasks or to allow the surgeon to concentrate to key actions during surgery rather than be burdened with operation of an additional device. For medical procedures which can be planned ahead of time and executed in a reasonable predictable manner, robot operation follows a concept which is often referred to as "Surgical CAD/CAM" (in analogy to Computer-Aided Design and Computer-Aided Manufacturing known from industrial manufacturing systems). One example is in the area of interventional radiology, where robots have the potential to position a needle guide, based on pre-operative planning in order to support the physician to push the needle through this guide. As imaging, tissue modeling, and needle steering technology improve, future systems are likely to become more highly integrated and will actively place the needles and therapy devices along paths that cannot be achieved by simply aiming a needle guide.

\subsection{State-of-the-Art in Medical Robotics}

Literature shows a couple of developed setups - and products - which have also been used clinically with moderate success. There is only a small number of commercial companies selling medical robots and thus the total number installed is relatively small (and dominated by one particular setup) [9]. Unlike the area of factory robotics, which grew rapidly during the 1970s and 1980s, Medical Robotics has not yet reached a critical mass. However, it is believed that the benefits of medical robots will become increasingly clear, which finally will lead to a continued rise of their use in clinical routines.

Some robots function as surgical assistants in orthopedics, and others can be used as a surgeon's "third hand" for moving the camera during minimally invasive procedures. Others exist to perform or facilitate tele-surgery, tele-monitoring, telementoring, or true tele-presence instruction. Still other robotic devices perform or 
assist with image guided interventions. Further possible classifications of surgical robots can be based on their level of autonomy as in:

- Passive - In which the robot serves as a guide to the surgeon's hands;

- Hands-on - In which the surgeon operates the device in the surgical field, but where the robot controller supports the surgeon and/or helps to avoid wrong actions ("shared control");

- Tele-operated - In which the robot is in the surgical field but is explicitly controlled by a surgeon some distance (usually a few feet, i.e., within the operating room) away;

- Automated - In which the robot executes pre-operatively planned trajectories;

or based on surgical application fields (Neurosurgery, Orthopaedics, Urology, Maxillofacial, Radiosurgery, Ophthalmology, Cardiac Surgery, Interventional Radiology) [3].

The area of image guided robots has been one of the starting points for medical robot systems, with applications in orthopedic surgery (e.g., ROBODOC, CASPAR, SPINEASSIST, RIO) and for neurosurgical applications (e.g., MINERVA, NEUROMATE, ROSA). Very close to the aforementioned systems are robot setups for interventional radiology (e.g., PAKY+RCM, B-ROB, INNOMOTION, MAXIO, ISYS 1) and external radiotherapy (e.g., CYBERKNIFE). Another group of robots is the one of "hands-on" and/or remotely controlled ("master/slave") setups like RAMS, MICRON for microsurgery, DAVINCI, ALF-X or camera holding assistants like AESOP, FREEHAND, SOLOASSIST, VICKY for laparoscopic surgery, and MAGELLAN for robot-guided catheter placement. A third category includes intracorporeal robot systems but only a few prototypes are described in literature at present (e.g., HEARTLANDER, EMUL).

Several databases are available via the Internet and provide a good overview about past and present medical robotic systems, like the "Surgical Robotics Blogspot" (http://surgrob.blogspot.com/).

\section{Open Research Questions}

It should be mentioned at this point, that only very few of the systems developed so far are being used in clinical routines. The main reason for this lack of success is not related to technical functioning, but to other factors, such as:

- Cumbersome use of robots (complexity, size, missing integration into clinical workflow) which hinders application in clinical routine; 
- High cost for a robot system and operational cost, i.e., cost/benefit ratio is not satisfactory, in most cases;

- High setup time and effort (e.g., need for an additional person to operate the robot system);

- Limitation in portability and/or mobility;

- Unsolved safety issues.

To demonstrate the potential benefits of medical robotic systems future research is required in both areas development of system components and the development of systems as a whole. In terms of system components, research topics can be outlined as follows.

System Architecture - For Medical Robotics to evolve as an independent field and to decrease the cost and difficulty of developing prototype systems, the establishment of a suitable system architecture is an enabling step. Development in systems architecture should emphasize modularity in mechanical design, control system electronics, and software. Middleware concepts such as ROS [10], OROCOS [11] for robot systems and IGSTK, 3DSLICER/PLUS [12] and others for image-guided surgical setups in broader context help for the prototyping phase - extension of such concepts toward development of medical products still needs to be investigated in more detail.

Software Design - The development of a software environment for Medical Robotics is a significant challenge. Research should be based - along with the system architecture mentioned above - on software frameworks geared to the medical environment and especially considering safety and robustness. While this software environment would still need to be customized for different surgical procedures, research on new applications would at least have a better starting point for the development work.

Mechanical Design - Novel mechanical designs are needed to improve the utility of robotics in medical procedures. It is the author's belief that special purpose mechanical designs are more appropriate for most applications. In particular, these designs should be safer, as they can be designed specifically for the medical environment and customized for different medical procedures. However, it should be noted that special purpose designs will not enjoy the same economies of scale as more general designs, which should be addressed by developing a set of reusable and configurable modules for dedicated designs.

Compatibility to Imaging Devices - With the increasing popularity of imageguided interventions, robotic systems need to be able to work within the constraints of various clinical imaging modalities. While these systems currently are still under the direct control of the physician most of the time, in the future they will increasingly be linked to these imaging modalities. 
Safety Issues - Safety is a paramount concern in the application of a medical device and must be addressed appropriately to move the field forward. Safety issues have been discussed by many authors (e.g., [4] and [5]) - all of them coming to the conclusion that Medical Robotics is a completely different application from industrial robots, e.g., because medical robots must operate in cooperation with, and even more important directly on humans to be fully effective. Therefore suitable safety levels must be defined and discussed by the community at large. This opens the question about what guidance is available for robotic developers in order to support the development process. Are there any best-practice examples for basic orientation? As a matter of fact, there are some standards in place, which define the safety requirements for medical devices. But interpretation of these requirements and transfer into concrete actions is rather complicated, especially for developers lacking long-term experience in the field. A dedicated standard for robots in personal care, in particular for medical applications, is in preparatory stage and should give more guidance for development of such devices.

User Interface - Known surgical robotic setups are mainly dominated by rigid interaction modes such as tele-operation (co-manipulation) or 'autonomous execution under supervision'. The gap between both procedures is large and the choice quickly is towards tele-operation when a certain level of complexity is reached by the application (e.g., tissue movement during intervention). Occasionally some hybrid forms - mostly featuring pre-operatively determined additional guidance or constraints - can be encountered. But the many questions remain: What is a suitable user interface for a medical robot? The answer will certainly vary depending on the medical task for which the robot is being designed. It seems that medical robots will initially at least be more accepted by physicians if the physicians feel to be still in control of the entire procedure.

\section{Selected Examples for Medical Robotic Systems}

In the following some selected examples for medical robotic systems, in particular for needle-based procedures, are briefly described. All described systems have been developed under the supervision of the author and have already demonstrated their potential in practical use.

\subsection{Robot-Assisted Biopsy: B-Rob I and B-Rob II}

Basic problem for percutaneous interventions is to target a needle-based instrument through the skin and thus without direct sight exactly to the region of interest (e.g., a tumor). Different types of intra-operative imaging modalities, like ultra-sound imaging (US), computed-tomography (CT) or magnetic resonance 
tomography (MR) are being used in order to support the procedure. Compared to a traditional (freehand) technique, robotics assistance potentially helps to improve the procedure. Such a robot could serve as the physician's "third hand", exactly maintaining the previous planned trajectory and (sometimes; especially in case of deeply situated target areas) helping to shorten the time associated with definition of the desired insertion point and angulation.

Prototype B-Rob I. Starting in 2001, a custom made robotic targeting device for interventional radiology - called B-Rob I - was developed by ARC Seibersdorf Research GmbH (Seibersdorf, Austria) together with the Departments of Diagnostic Radiology and of Biomedical Engineering and Physics, Vienna Medical University (Vienna, Austria). The main goal of the system was to improve diagnostic accuracy as well as to reduce the risk of possible complications while planning of an oblique trajectory. Planned clinical applications for this first prototype have been biopsies in the abdominal area using US and CT as intra-operative imaging modalities.

The planned use of the system with different CT-scanners as well as with USsystems led to a tailored 7 DOF (degrees-of-freedom) kinematic structure of the robot system. Delivery to the skin entry point was performed by a 4 DOF gross positioning system consisting of three linear axes in Cartesian configuration together with one additional rotational link. Final orientation of the needle was done by means of a dedicated "Needle Positioning Unit" (NPU) with its two linear DOFs. Another linear DOF with a limited stroke of $50 \mathrm{~mm}$ was moving the entire NPU to the patient's skin in a secure approach movement, i.e., with minimized velocity and force. Setting the needle orientation was strictly decoupled from movement of any axis of the gross positioning system. Remote center of motion ("pivot point") for angulation of the needle was maintained by the kinematic structure of the NPU in order to achieve the maximum of safety during the intervention. Registration of the robot system to the imaging space, as well as, measurement of the current patient position was performed by an optical tracker system (Polaris, NDI, Canada).

Performance of the complete system was evaluated in a series of in-vitro tests using a needle-penetrable phantom. Peas (diameter $=9.4 \pm 0.7 \mathrm{~mm}$ ) were embedded within a custom-made gel-phantom, as targets. Biopsy needles were inserted according to a previous planning procedure and distance between actual needle tract and center of the target was evaluated. During this in-vitro study, the robot system could demonstrate highly sufficient accuracy for the medical application in question. Each of the biopsy targets ( $n=40$ for US-guidance; $n=20$ for CT-guidance) could be reached straightaway. Radial deviation between the center of the target and the needle tract was measured with $2.18 \mathrm{~mm} \pm 1.25 \mathrm{~mm}$ for US-guided interventions and $1.48 \mathrm{~mm} \pm 0.79 \mathrm{~mm}$ for CT-guidance. [6] 
Prototype B-Rob II. Apart from the very promising results concerning accuracy, in-vitro trials with B-Rob I have also shown that size and complexity of the setup would be a major barrier for use in clinical routine. A new prototype - called BRob II - thus should transfer the proofed concepts from B-Rob I into a practical clinical setup. Development started in 2004 in a cooperation between PROFACTOR Research and Solutions GmbH (Seibersdorf, Austria) and Medical Intelligence $\mathrm{GmbH}$ (Schwabmünchen, Germany) with the major goals to create a modular setup of a robot assistant for a broad variety of clinical applications as well as to significantly reduce the technical complexity of the system in order to reach an acceptable cost/benefit ratio for clinical application.

The new B-Rob II setup consisted of two 2DOF positioning modules with small size (dimensions of one 2DOF module: $\mathrm{WxLxH}=100 \mathrm{~mm} \times 150 \mathrm{~mm} \times 30 \mathrm{~mm}$ ). Connection of the two modules with two carbon "fingers" - forming a parallelogram structure - allowed needle angulation of $\pm 30^{\circ}$ and $2 \mathrm{DOF}$ positioning of $\pm 20 \mathrm{~mm}$ with high accuracy (see Figure 1, left). For efficient gross positioning of the robot system, the modules were mounted on a passive 7DOF multi-functional holding arm (ATLAS Arm, Medical Intelligence GmbH).

A series of in-vitro studies was performed with B-Rob II, with a similar setup as described above. 50 biopsy procedures were successfully performed showing a calculated average targeting error of $1.05 \mathrm{~mm} \pm 0.35 \mathrm{~mm}$ [7]. The design goal of providing with a modular setup could be achieved and demonstrated in different application setups. In cooperation with Johns Hopkins University (Baltimore, USA) the robot, after slight modification to extend the reach of motion from \pm 2 $\mathrm{cm}$ in $\mathrm{x}$-y-plane to $\pm 4 \mathrm{~cm}$, has been configured for TRUS-guided brachytherapy of the prostate with very promising results, including a clinical study with 10 patients (see Figure 1, right). [13]
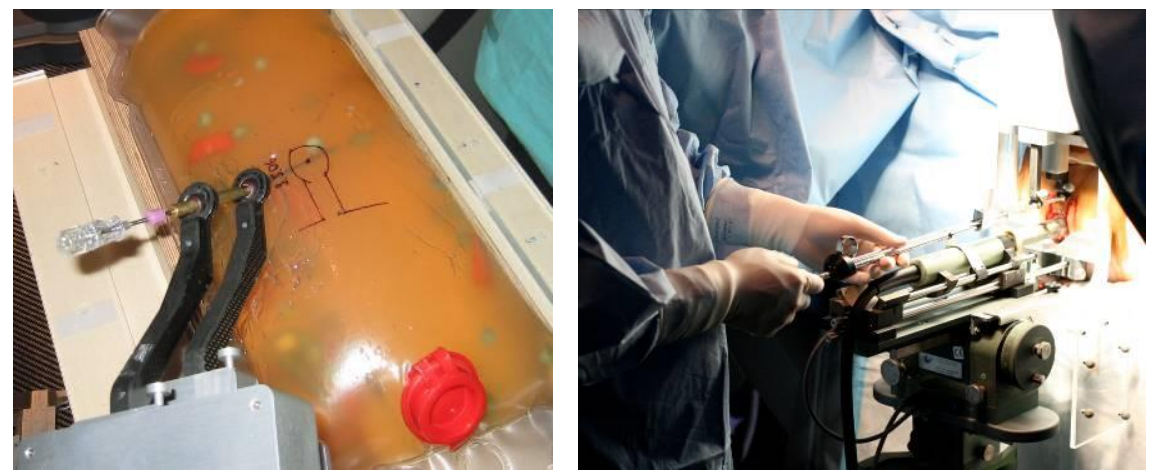

Figure 1

Robot system B-Rob II during in-vitro trials; Modified B-Rob II with extended range of motion in clinical trial for TRUS-guided prostate biopsy (courtesy of ACMIT Gmbh and Johns Hopkins University) 
Together with the ENT department of University of Erlangen (Erlangen, Germany) the robot, after slight modification to extend the robot's payload, has been evaluated as camera holder for trans-nasal endoscopic applications (see Figure 2, left). [14] Together with two clinical partners - neurosurgical department of Wagner-Jauregg hospital in Linz (Linz, Austria) as well as neurosurgical department of Krankenhaus Karlsruhe (Karlsruhe, Germany) - the robot system also has been successfully evaluated for brain-biopsy applications (see Figure 2, right). [15]

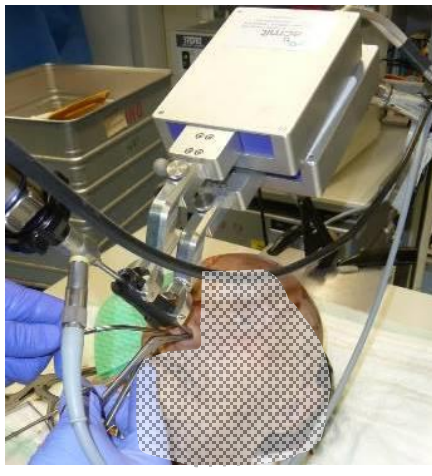

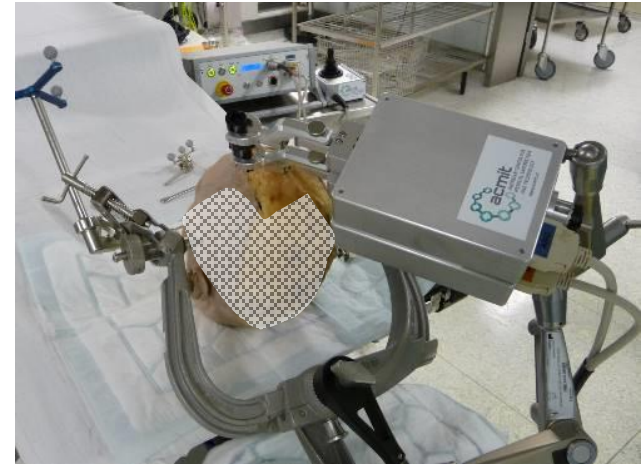

Figure 2

Modified B-Rob II with extended payload in cadaver trials for the use as tool holder in ENT and neurosurgery (courtesy of ACMIT Gmbh, Austria)

\subsection{Robot System iSYS 1}

In close cooperation with the iSYS Medizintechnik GmbH (Kitzbühel, Austria) the B-Rob II robot system has been redesigned for use as remote-controlled needle holder for interventional radiology. Dedicated workflow and setups for two imaging modalities, cone-beam CT (CBCT) and classical computed tomography imaging (CT), have been developed and have successfully passed CE certification.

Similar to the B-Rob II concept, this robot setup consists of a 7 DOF passive "Multi-Functional Arm" (MFA) for pre-positioning of the 2x2 DOF active "Robot Positioning Unit" (RPU). The general kinematic concept for the active robot is based on a parallelogram mechanism, with two parallel "Needle Guide Extensions" (NGE) showing relative linear movement and being connected to each other by means of a dedicated "Needle Guide Adapter" (NGA). The applied highly integrated design results in a small footprint of the robot (WxLxH: $120 \mathrm{~mm}$ x $200 \mathrm{~mm}$ x $70 \mathrm{~mm}$ ) which allows the use of the system also inside the CT-gantry without kinematic restrictions. The range of motion (ROM) for the active robot results in $+/-20 \mathrm{~mm}$ for translation in a $\mathrm{x}$-y-plane (for fine adjustment of the needle entry point) and $+/-30^{\circ}$ for needle angulation. The use of high precision 
components for the mechanical system (anti-backlash gear stage, high-precision ball screw system and monorail guides, etc.) results in a positioning resolution of about $5 \mu \mathrm{m}$ and/or an angulation resolution of about 1/100 degree. For guidance of the tool, i.e., the needle, a set of "Needle Guide Inserts" (NGI) is available for the NGA. With these NGIs it is possible to have precise guidance of the needle during insertion, and release it from the robot's NGA, by pulling out the NGI and opening it. Beside of the RPU and the MFA, the robot setup consists of a set of adapters in order to securely mount the MFA to the patient supporting table ("Table Top Adapter" - TTA). A "Control Unit" (CU) and a dedicated "Human Control Unit" (HCU), both of which prepared for attachment to the side rail of the table (see Figure 3, left), complete the setup.

"Registration" of the robot to the acquired images in general requires a structure which can be easily and stably identified in the pre-/intra-operative images as well as in robot coordinates. For the CBCT-workflow, a "Needle Guide Marker Insert" (NGMI) has been designed which includes two small metal rings in line with the needle insertion axis. By moving the imaging plane of the CBCT into a "bull's eye view" direction (i.e., into direction of the trajectory) the NGI can be exactly aligned according to the planned needle trajectory by remotely bringing the aforementioned rings into concentric position. For the available CT-workflow, two dedicated marker structures, equipped with $10 \mathrm{~mm}$ glass balls in exactly known positions, are mounted on the robot. After acquisition of a 3D data set (which is also used to plan the needle trajectory), a fully automatic registration process of the dedicated planning software "RoboNav" (iSYS Medizintechnik $\mathrm{GmbH}$, Austria) segments the registration marker and exactly calculates the robot position relative to the patient.
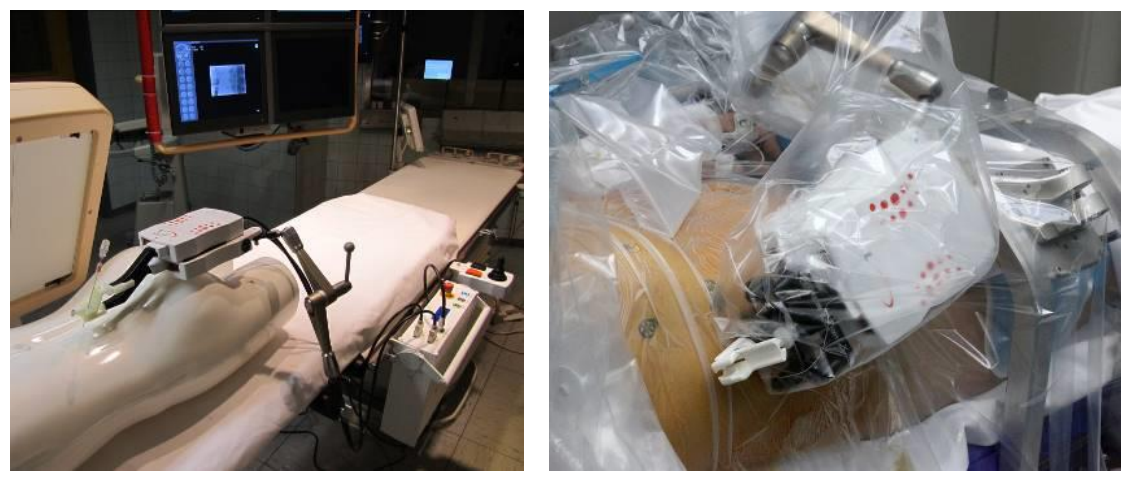

Figure 3

Robot system "iSYS 1" in CBCT configuration and in CT configuration in clinical setup (courtesy of iSYS Medizintechnik GmbH, Austria)

The iSYS 1 robot system now is in routine clinical use in several hospitals around the world with applications ranging from classical biopsy procedures over tumor ablation with different procedures (RFA, IRE, microwave, cryo-ablation) to 
orthopedic procedures (see Figure 3, right). In addition, a patient study in cooperation with the Institute for Neurosurgery of the Medical University Vienna (Vienna, Austria) is currently in progress in order to evaluate the suitability of the iSYS 1 robot system for a set of neurosurgical procedures [16].

\subsection{VIRTOBOT - Robot System for VIRTOPSY Procedures}

The concept of Virtopsy was born from the desire to implement new techniques in digital imaging (CT/MR), three dimensional (3D) surface scanning, photogrammetry, and post-mortem minimally invasive biopsy for the benefit of forensic science. The aim is to establish an observer independent, objective and reproducible forensic assessment method with digitally storable results. In order to support such procedures a robotic system, called VIRTOBOT, was developed in cooperation with the Institute for Forensic Medicine in Zurich (Zurich, Switzerland). The entire system consists of a Multi-Slice CT scanner (Somatom Emotion 6, Siemens, Germany), a modified heart-lung machine for post mortem angiography, as well as, the VIRTOBOT robotic system which operates different system tools. Additionally, a high precision optical tracking system (Polaris, NDI, Canada) is integrated to increase the placement precision of automatic tissue sampling.

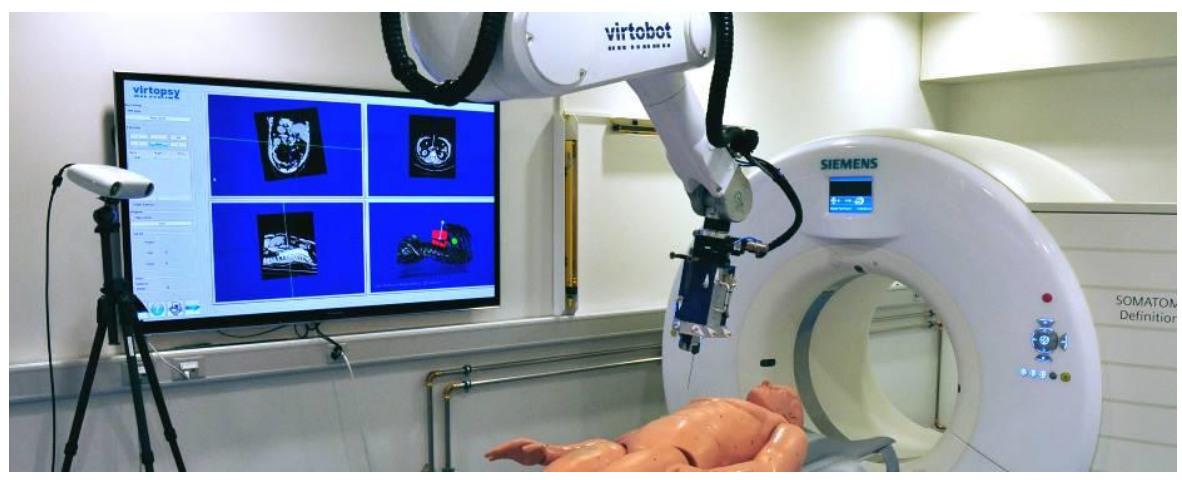

Figure 4

VIRTOBOT system on biopsy configuration (courtesy of IRM Zurich, Switzerland)

The VIRTOBOT comprises a Stäubli TX90L robotic arm and a $4 \mathrm{~m}$ external linear axis mounted on the ceiling in parallel to the CT table axis. Several safety precautions have been implemented into the VIRTOBOT system cell beside the typical emergency stop switches. Safety light curtains (Safety Class 4) ensure redundant safety during robot operation by triggering an immediate emergency stop of the complete system when being interrupted. Two further safety light curtains (Safety Class 2) are mounted between the robot tool and the CT-scanner in order to avoid collisions with the imaging system. 
Complete workflow of the system and coordination of the application software packages (navigation and planning software, surface scan and photogrammetry software, robot control) is organized by the VCC software (Virtopsy Control Center). The VCC links diagnostic information and following process data of photogrammetry and surface scanning with the acquired CT scan dataset and stores the information in a "session". The session manager collects all data and documents for the whole process of a particular Virtopsy procedure.

The VIRTOBOT system has been in routine use since 2014. Use of the system helps in minimizing errors and leads to better and more reproducible results. Compared to the manual procedure, overall scanning times can be reduced significantly [8].

\section{Conclusions}

Among medical robotic systems, mechatronic assistants for percutaneous procedures show a high potential. Such robots help guide the needle into the most promising region of the lesion with a very high accuracy. As a consequence, more efficacious characterization and treatment, particularly for lesions that are difficult to target, can be anticipated.

\section{Acknowledgements}

The author thanks the development team at ACMIT (Martin Fürst, Martin Kornfeld, Wolfgang Ptacek, Nikolaus Hocke, Peter Mainer) as well as our cooperation partners Johns Hopkins University (Peter Kazanzides), Queens University (Gabor Fichtinger), Krankenhaus Karlsruhe (Uwe Spetzger), IRM Zurich (Michael Thali), and iSYS Medizintechnik GmbH (Michael Vogele).

\section{References}

[1] Davies B: A Review of Robotics in Surgery, Proceedings of Inst. Mechanical Engineering, 214, 129-140 (2000)

[2] Howe RD, Matsuaka Y: Robotics for Surgery, Annu. Rev. Biomed. Eng., 1, 211-240 (1999)

[3] Moore JE, Zouridakis G: Biomedical Technology and Devices Handbook, The Mechanical Engineering Handbook Series, 32-1-32-24, CRC Press (2004)

[4] Davies B: A Discussion of Safety Issues for Medical Robotics, Journal Computer-Integrated Surgery, Taylor. R. H. et al. (Eds) 287-296, MIT Press, Cambridge (1996)

[5] Elder MC, Knight JC: Specifying User Interfaces for Safety-Critical Medical Systems, Journal Medical Robotics and Computer Assisted Surgery, 148-155, Wiley (1995)

[6] Kettenbach J, Kronreif G, Figl M, Fürst M, Birkfellner W, Hanel R, Ptacek $\mathrm{W}$, Bergmann $\mathrm{H}$ : Robot-assisted Biopsy Using CT-Guidance: Initial 
Results from In-Vitro Tests, Journal Investigative Radiology, Volume 40, Issue 4, 219-228, Lippincott Williams \& Wilkins (2005)

[7] Kronreif G, Fürst M, Ptacek W, Kornfeld M, Kettenbach J: Robotic Platform B Rob II: In-vitro Tests and Results, In: $4^{\text {th }}$ International Conference on Computer Aided Surgery around the Head, Innsbruck (2007)

[8] Ebert L, Ptacek W, Naether S, Fürst M, Ross S, Buck U, Weber S, Thali M: Virtobot -- a Multi-Functional Robotic System for 3D Surface Scanning and Automatic Post Mortem Biopsy, Journal Medical Robotics and Computer Assisted Surgery, 6(1), Wiley, 18-27 (2010)

[9] Hoeckelmann M, Rudas IJ, Fiorini P, Kirchner K, Haidegger T: Current Capabilities and Development Potential in Surgical Robotics, International Journal of Advanced Robotic Systems, Vol. 12:61, ISSN 1729-8806 (2015)

[10] ROS - Robot Operating System: http://www.ros.org/ (last visit: Dec 2015)

[11] Open Robot Control Software - OROCOS: http://www.orocos.org/ (last visit: Dec 2015)

[12] 3DSLICER: https://www.slicer.org/ (last visit: Dec 2015)

[13] Song DY, Burdette EC, Fiene J, Armour E, Kronreif G, Deguet A, Iordachita I, Fichtinger G, Kazanzides P: Robotic Needle Guide for Prostate Brachytherapy: Clinical Testing of Feasibility and Performance, Brachytherapy, 10(1):57-63 (2011)

[14] Bohr C, Milojcic R, Kronreif G, Fuerst M, Stegman A, Vogele M, Wurm J, Iro H: Feasibility Study for a New Robot Design, B-Rob II, in Lateral Skull Base and Transsphenoidal Surgery, Proc. $5^{\text {th }}$ World Congress for Endoscopic Surgery of the Brain, Skull Base \& Spine (2012)

[15] Kronreif G, Ptacek W, Kornfeld M, Fürst M: Evaluation of Robot Assistance in Neuro-Surgical Applications, Journal of Robotic Surgery, Volume 6, Issue 1, pp. 33-39 (2012)

[16] Kronreif G, Ptacek W, Fürst M, Vogele M: iSYS 1 Robot System for Neurosurgical Applications, Abstract book, DGNC 2015 (2015) 